\title{
Formation control for multiple unmanned aerial vehicles in constrained space using modified artificial potential field
}

\author{
Hang Yin*, Léa L. Cam, Utpal Roy \\ Department of Mechanical \& Aerospace Engineering, Syracuse University, NY, USA \\ Email: hayin@syr.edu
}

\begin{abstract}
This paper addresses a formation tracking control issue for multiple Unmanned Aerial Vehicles (UAV) flying through a constrained space. Based on the Formation Potential Field (FPF), a Modified Artificial Potential Field (MAPF) technique is proposed, which guarantees to generate and maintain a given formation while avoiding collisions. The technique has two phases. UAVs are gathered around the formation center first during Phase 1, and the formation is then achieved in Phase 2. Furthermore, an obstacle repulsive potential field is introduced into this approach to deal with collision avoidance under environmental constraints. By doing so, discontinuities of the original Formation Potential Field can be avoided, and the stability of formation generation could be enhanced. Simulation results are presented to illustrate the validity of proposed approach.
\end{abstract}

Keywords: Formation Control, Collision Avoidance, Artificial Potential Field, UAV.

\section{INTRODUCTION}

A significant amount of research efforts has been reported on the control of multi-agent systems to both their practical potential in various applications and theoretical challenges arising in coordination and control of those agents. Formation control, which is one the most actively studied topics within the realm of multi-agent systems, generally aims to drive multiple agents to achieve given states. Recently, formation control of unmanned aerial vehicle (UAV) systems has drawn considerable attention from the scientific and engineering communities. Many interesting applications of formation flying have been studied. Examples include forest fire monitoring [1] and radar deception [2]. The Artificial Potential Field (APF) approach on formation control of Multi-Agents System (MAS) becomes one of the most popular techniques.

The APF approach was first introduced by Khatib [3]. It was presented in the context of collision avoidance. The basic concept of the APF method requires filling the activity area (the whole flying envelope of the UAVs) with the artificial potential field in which the agent is attracted to its target position, and is repulsed away from the obstacles and other agents [4]. This method is attractive because of its elegant mathematical formulation and simplicity. In the following decades, this method is extended to address autonomous agent path planning either in stationary environments, where target and obstacles are not moving, or in dynamic environments [4] [5] [6]. In recent years, researchers start applying the APF technique in formation control of MAS. For example, a method combining the APF technique with Kane's method has been proposed to solve the multi-hybrid-driven underwater glider motion planning and obstacle avoidance problem [7]. Similarly, Asl et al. [8] has used another technique, called Asexual Reproduction Optimization, which is based on the APF and is combined with the leader-follower formation method to formulate the path planning problem. However, the spatial constraints were not considered in most of the previous works.

Another significant work, Liu et al., proposes a novel Formation Potential Field (FPF) method for multi-agents' formation problem, which is designed combining multiple local attractive potential fields with multiple local repulsive potential fields. A global attractive potential is added to guide agents which are away from the attractive regions of a local attractive potential. Additionally, a collision avoidance strategy, which is based on the Artificial Potential Field and Dirac delta function, is applied [9]. This strategy locally modifies the original trajectory of a virtual leader, which is always located in the formation center, so that all agents in this formation will be able to avoid collisions when unexpected special constraints are detected while maintaining a given formation. Furthermore, the auxiliary design system [9] [10], is designed to impart stability to the dynamic formations during the process trajectory tracking when the "saturation effect" of inputs are considered.

However, there are two drawbacks of this method. First, the added global attractive potential field has a disrupting effect on the smoothness of the whole potential field. In other words, gradients at some specific points of this field may or may not exist due to the presence of this global attractive potential, as 
it is shown in Figure 1. These discontinuities of the formation potential field may cause abrupt accelerations of the UAVs which may have a negative effect on the overall system stability. Though this negative effect can be reduced with the auxiliary design system, it can't be eliminated.

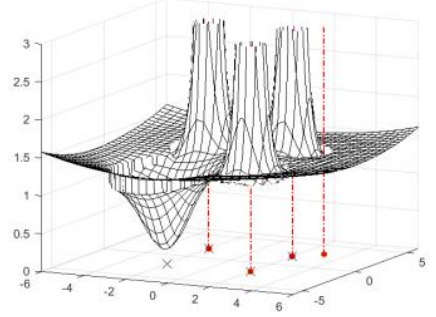

Figure 1. Formation potential field method failure case

Second, the FPF method can still fail to achieve its goal when agents are not well distributed around the formation area. Figure 1 also shows a sample case that one agent (marked with a red dot on the right side) can't find its way to the target position (marked with a black cross on the left) since the influence of a local attractive potential has been blocked by other three agents.

Inspired by above limitations, a Modified Artificial Potential Field (MAPF) method has been proposed in this paper to control multiple UAVs maintaining a given formation. Like the original FPF method, collisions between UAVs or between an UAV with other obstacles can be avoided by using this MAPF technique. Each UAV can be attracted to the target position without further guidance. The technique can deal both static or dynamic obstalces. The main difference between the proposed MAPF method and the FPF technique is that: (i) the Modified Artificial Potential Field can avoid situations where other agents' repulsive potential field neutralize the attractions of the target attractive potential field when it should not; and (ii) the negative effect of the FPF discontinuities can be prevented.

\section{PROBLEM STATEMENT}

The formation keeping problem of multiple UAVs in a dynamic environment consist of three problems: (i) how to plan, control and generate a given UAVs formation at their initial positions; (ii) moving UAVs as a group in formation from the initial position to the destination; (iii) avoidance of collisions during their flight. To simplify the analysis, we make the following definitions and assumptions:

Definition 1 (Fixed Formation [11]): A formation pattern is defined as a fixed formation if $\Delta \boldsymbol{x}_{i}(t)=\boldsymbol{x}_{i}^{d}(t)-\boldsymbol{x}^{r}(t), i=$ $1, \ldots, n$, remains time-invariant for $t \in[0, \infty)$. It means

$\Delta \dot{\boldsymbol{x}}_{i}(t)=\dot{\boldsymbol{x}}_{i}^{d}(t)-\dot{\boldsymbol{x}}^{r}(t)=0$

where $\boldsymbol{x}_{i}(t)$ is the position of the UAV, $v_{i}$, at time $t ; \boldsymbol{x}_{i}^{d}(t)$ is the desired position of the UAV, $v_{i}$, at time $t ; \boldsymbol{x}^{r}(t)$ is the center of the desired formation at time $t$.

$\boldsymbol{x}^{r}(t)=\frac{1}{n} \sum_{1}^{n} \boldsymbol{x}_{i}^{d}(t)$
Assumption 1: An UAV is able to estimate its position in the global coordinate system.

Assumption 2: Each UAV has the capability to communicate with other UAVs anytime and anywhere.

Based on above definition and assumptions, the control goal is: design a control strategy for multiple UAVs, which are initialized at random positions, and then generate the given formation while avoiding collisions. The control goal can be expressed mathematically as follows [9]:

$\sum_{i=1}^{n}\left\|\tilde{x_{l}}(t)\right\| \leq \varepsilon,\left\|x_{0}(t)-\boldsymbol{x}^{r}(t)\right\| \leq \epsilon$

where $\varepsilon$ and $\epsilon$ are two small constants; $\widetilde{\boldsymbol{x}}_{l}(t)$ defined as the tracking error of UAV $v_{i}$ at time $t . \boldsymbol{x}_{0}(t)$ is the location of the virtual leader.

$\widetilde{\boldsymbol{x}_{l}}(t)=\left\{\min \left(\left\|\boldsymbol{x}_{i}(t)-\boldsymbol{x}_{j}^{d}(t)\right\|, j=1, \ldots, n\right)\right\}$

$\boldsymbol{x}_{0}(t)=\frac{1}{n} \sum_{1}^{n} \boldsymbol{x}_{i}(t)$

\section{MODIFIED ARTIFICIAL POTENTIAL FIELD}

\subsection{Global task function and local task function}

There are two kinds of tasks defined in this paper: (i) global tasks and (ii) local tasks. A potential function will be assigned to each of the task and the function which has an effect on either global or local.

The purpose of the global attractive potential is: attracting an UAV to the target position wherever the UAV is. The function should have effect on the coordinate system without singular points. Therefore, the global attractive task function defined as follows:

$f_{g a}\left(\boldsymbol{x}, \boldsymbol{x}^{S}\right)=\frac{1}{2}\left(\boldsymbol{x}-\boldsymbol{x}^{S}\right)^{T} K\left(\boldsymbol{x}-\boldsymbol{x}^{S}\right)$

$\boldsymbol{x}^{\boldsymbol{s}}$ is the location of a potential source, also known as the target location $K$ is a weight matrix which assign different weight on each dimension.

Unlike the global attractive task, the local attractive task focuses on attracting UAVs within a relatively small range. In order to satisfy this requirement, the local attractive function should decay quickly. Hence, the local attractive task function is chosen to be:

$f_{l a}\left(\boldsymbol{x}, \boldsymbol{x}^{S}\right)=-\exp \left[-\frac{1}{2}\left(\boldsymbol{x}-\boldsymbol{x}^{S}\right)^{T} K\left(\boldsymbol{x}-\boldsymbol{x}^{S}\right)\right]$

The main purpose of a local repulsive task is to avoid collisions between two UAVs or between an UAV with its environment (environmental constraints). The local repulsive task is written as:

$f_{l r}\left(\boldsymbol{x}, \boldsymbol{x}^{S}\right)=\frac{1}{2\left(\boldsymbol{x}-\boldsymbol{x}^{S}\right)^{T} K\left(\boldsymbol{x}-\boldsymbol{x}^{S}\right)}$

\subsection{Formulations of the modified artificial potential field}

Two separate phases are designed to generate and keep the given formation all alone formation trajectory. In Phase 1, randomly located UAVs will be gathered around the given formation center, and it will make sure all UAVs are well distributed. When all UAVs surround the formation center, 
Phase 2 will start. In this phase, each UAV will find its own way to its desired position without further guidance.

In Phase 1, the main purpose of the global attractive potential field is to gather UAVs into one place, as shown in Figure 2. Therefore, the global attractive potential field for any $\mathrm{UAV}_{i}$ is defined as:

$U_{i}^{g a}\left(\boldsymbol{x}_{i}, \boldsymbol{x}^{r}\right)=k_{a 1} f_{g a}\left(\boldsymbol{x}_{i}, \boldsymbol{x}^{r}\right)$

where $k_{a 1}$ is a positive constant. Please note that in Phase 1, the center of the global attractive potential is always at the same position with the desired formation, $\boldsymbol{x}^{r}$.

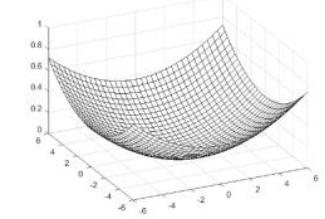

Figure 2. Global attractive potential field

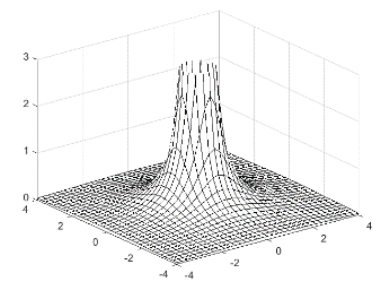

Figure 3. Local repulsive potential field

The purpose of the local repulsive potential is to guide UAVs in a well distributed fashion around the formation center. The local repulsive potential in Phase 1 can be written as:

$U_{i}^{l r}\left(\boldsymbol{x}_{i}, \boldsymbol{x}^{r}\right)=k_{r 1} f_{l r}\left(\boldsymbol{x}_{i}, \boldsymbol{x}^{r}\right)$

where $k_{r 1}$ is a positive constant.

Notice, the ratio between $k_{a 1}$ with $k_{r 1}$ will affect how far away UAVs will be distributed around the given formation center. How to select these two values of $k_{a 1}$ and $k_{r 1}$ will be discussed in following section.

By combining one global attractive potential with one local repulsive potential, we obtain the Phase 1 potential field, which is defined as follows:

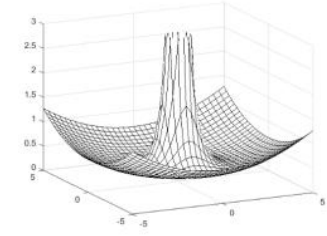

Figure 4. Potential field for Phase 1

$U_{i}^{1}=U_{i}^{g a}+U_{i}^{l r}$

The Phase 2 potential field is constructed with the local attractive potential fields and local repulsive potential fields. For a random $\mathrm{UAV}_{i}$, local attractive potentials are located in desired formation positions and centers of local repulsive potentials are at other UAVs' locations. Thus, the local attractive potential field for the $\mathrm{UAV}_{i}$ is:

$U_{i}^{l a}\left(\boldsymbol{x}_{i}, \boldsymbol{x}^{d}\right)=1+k_{a 2} \sum_{j=1}^{N} f_{l a}\left(\boldsymbol{x}_{i}, \boldsymbol{x}_{j}^{d}\right)$

Like $k_{a 1}, k_{a 2}$ is a positive constant. $\boldsymbol{x}_{j}^{d}$ is one of the desired formation locations, which treat as potential source $j$.

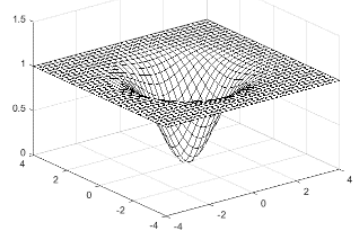

Figure 5. Local attractive potential field

Theoretically, the value of $U_{i}^{l a}$ can be as small as it could be. The smaller the minimum value of $U_{i}^{l a}$, the bigger the attractive area. In order to avoid the negative affect of two overlapping local attractive potential fields, another assumption has to be made to guarantee proper distance between any two desired formation positions.

Assumption 3: The desired formation satisfies the condition that for any UAV position $\boldsymbol{x}$ in the space $U_{i}^{l a} \geq 0$ [9].

The other part of Phase 2 potential field is the summation of a group of local repulsive potential fields, which is generated by other UAVs. It can be written as follows:

$U_{i}^{l r}=k_{r 2} \sum_{j=1, j \neq i}^{N} f_{l r}\left(\boldsymbol{x}_{i}, \boldsymbol{x}_{j}\right)$

where $k_{r 2}$ is a positive constant; $\boldsymbol{x}_{i}$ is the location of UAV $v_{i}$; and $\boldsymbol{x}_{\boldsymbol{j}}$ is the location of UAV $\boldsymbol{v}_{j}, j \neq i$.

By adding (12) and (13) together, we obtain the potential field for Phase 2.

$U_{i}^{2}=U_{i}^{l a}+U_{i}^{l r}$

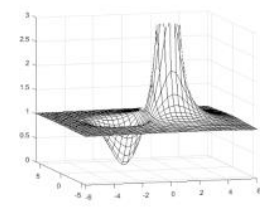

Figure 6. Potential field for Phase 2

The criteria for moving of the UAVs' formation from Phase 1 to Phase 2 is critical. Two necessary conditions are: (i) are UAVs must gather around formation area first, (ii) the UAVs must well distributed. To satisfy above two requirements, the following conditions are proposed:

$\left\{\begin{array}{l}\text { for Phase }=2, \quad \sigma>\sigma_{0} \text { and } \frac{\bar{d}_{\cdot r}}{\lambda}>\varepsilon \\ \text { for Phase }=1, \text { otherwise }\end{array}\right.$

where $\sigma_{0}$ and $\varepsilon$ are small value constants; $d_{i j}$ is the distance between $\mathrm{UAV}_{i}$ and $\mathrm{UAV}_{j} ; \bar{d}_{\cdot r}$ is the average distance between UAVs with the virtual leader; $\lambda$ is the radius of the minimum circle, or sphere that is able to cover the whole desired 
formation, and $\sigma$ is the standard deviation of minimum distances between UAVs. They are defined as:

$\sigma=\sqrt{\frac{1}{n}\left[\left(\min \left(d_{1}\right)-\bar{d}\right)^{2}+\cdots+\left(\min \left(d_{n}\right)-\bar{d}\right)^{2}\right]}$

$\bar{d}=\frac{\min \left(d_{1} \cdot\right)+\min \left(d_{2} \cdot\right)+\cdots+\min \left(d_{n \cdot}\right)}{n}$

$\min \left(d_{1}\right)$ is the minimum distance between $\mathrm{UAV}_{i}$ with other UAVs.

Therefore, the Modified Artificial Potential Field can be written as:

$\bar{U}_{i}^{f o r}=\left\{\begin{array}{l}U_{i}^{1}, \text { if Phase }=1 \\ U_{i}^{2}, \text { if Phase }=2\end{array}\right.$

\subsection{Parameter optimization}

The ratios among $k_{r 1}, k_{a 1}, k_{r 2}$ and $k_{a 2}$ are critical, since these values will determine characteristics of the potential field. In this section, we will discuss how to optimize them.

It is not hard to show that, in Phase 1 the potential field $U_{i}^{1}$, the potential value reaches minimum when the point is located on a $2 \mathrm{D}$ base circle (2D case) or on a spherical surface (3D case). It is not hard to compute the circle's or the sphere's radius is $R=\sqrt{k_{r 1} / k_{a 1}}$. Since we'd like distribute UAVs around the given formation area, the radius of the minimum circle or sphere, $\lambda$ (which would be able to cover the whole desired formation), is a good reference value. Therefore, the ratio between $k_{r 1}$ with $k_{a 1}$ can be written as:

$k_{a 1} / k_{r 1}=1 / r \lambda^{2}$

where $r$ is a positive relaxation constant.

In Phase 2, the improper ratio between $k_{a 2}$ with $k_{r 2}$ will result in generating a local minimum region which will trap UAVs, and will prevent them moving forward to their desired locations. In order to avoid local minimum, the relationship between $k_{a 2}$ with $k_{r 2}$ has been studied in [9], and the suggested relationship is:

$k_{a 2} / k_{r 2}<0.4313$

\subsection{Collision avoidance}

Let $\Pi=\Pi_{1} \cup \Pi_{2} \cup \ldots \cup \Pi_{R}$ denotes a constrained space; $L_{r}$ denotes the edge of a region $\Pi_{r}, r=1 \ldots R$. Hence the repulsive potential field $U_{i}^{c}$, which generated by spatial constraints, can now be written as:

$U^{c}(\boldsymbol{x})=\sum_{r=1}^{R} \iint_{-\infty}^{\infty} \frac{k_{c r} \delta\left(\left\|\boldsymbol{x}-\boldsymbol{s}-k P_{r}(\boldsymbol{s})\right\|\right)}{\|\boldsymbol{x}-\boldsymbol{s}\|-s_{0}} d s_{1} d s_{2}$

where $\delta(x)$ is the Dirac delta function which is applied to gather the potential forces from all points on edges of spatial constraints (obstacles), and which satisfying the following condition:

$\boldsymbol{x}_{i}-\boldsymbol{s}=k P_{r}(\boldsymbol{s}), r=1, \ldots, R$

$\mathrm{k}_{\mathrm{cr}}$ is the spatial constraint factor; $k$ is a positive constant; $s_{0}$ is a small constant regarded as the safety distance to avoid collisions with constraints edges, and $P_{r}(\boldsymbol{s})$ is the perpendicular direction from a point $\boldsymbol{s}$ on $L_{r}$. The definition of $P_{r}(\boldsymbol{s})$ (in $3 \mathrm{D}$ space) is:

$P_{r}(\boldsymbol{s})=\left[\begin{array}{ll}\frac{\partial L_{r}}{\partial s_{2}} & -\frac{\partial L_{r}}{\partial s_{1}}\end{array}\right]^{T}$

\subsection{Controller design}

We assume $n$ number of UAVs with the similar dynamic characteristics. These characteristics can be described by the following practical nonlinear differential equation as:

$\dot{\boldsymbol{x}}_{i}=\boldsymbol{F}_{i}\left(\boldsymbol{x}_{i}\right)-\overline{\boldsymbol{F}}_{i}^{f o r}+\boldsymbol{G}_{i}\left(\boldsymbol{x}_{i}\right) \boldsymbol{u}_{i}$

where $\boldsymbol{F}_{i}\left(\boldsymbol{x}_{i}\right) \in \mathbb{R}_{m \times 1}$ is a nonlinear vector; $\boldsymbol{G}_{i}\left(\boldsymbol{x}_{i}\right) \in \mathbb{R}_{m \times m}$ is a smoothly nonsingular function matrix; $\boldsymbol{u}_{i}$ represents the control vector. Since the nonlinear characteristics of the nonlinear system are not the focus of this paper, we assume all UAVs are linear time-invariant systems. Thus, $\boldsymbol{F}_{i}\left(\boldsymbol{x}_{\boldsymbol{i}}\right)$ and $\boldsymbol{G}_{i}\left(\boldsymbol{x}_{\boldsymbol{i}}\right)$ can be considered as constant vectors, which rewritten as $\boldsymbol{F}_{i}$ and $\boldsymbol{G}_{i}$.

We choose the controller $\boldsymbol{u}_{i}$ as:

$\boldsymbol{u}_{i}=\boldsymbol{G}_{i}^{+}\left(-\boldsymbol{F}_{i}+\overline{\boldsymbol{F}}_{i}^{\text {for }}+\dot{\boldsymbol{x}}^{r}\right)$

where $\boldsymbol{G}_{i}^{+}$is the pseudo inverse of $\boldsymbol{G}_{i}$, which can be calculated by using singular value decomposition (SVD) method; $\dot{\boldsymbol{x}}^{r}$ is the virtual leader moving distance in unit time; $\overline{\boldsymbol{F}}_{i}^{\text {for }}$ is virtual force generated from potential field, defined as follows:

$\overline{\boldsymbol{F}}_{i}^{\text {for }}=\left\{\begin{array}{l}\boldsymbol{F}_{i}^{1}, \text { if Phase }=1 \\ \boldsymbol{F}_{i}^{2}, \text { if Phase }=2\end{array}\right.$

$\boldsymbol{F}_{i}^{1}=\boldsymbol{F}_{i}^{l r}+\boldsymbol{F}_{i}^{g a}=-\nabla U_{i}^{l r}-\nabla U_{i}^{g a}$

$=-2 k_{r 1} \frac{\partial f_{l r}\left(\boldsymbol{x}_{i}, \boldsymbol{x}^{r}\right)}{\partial x_{i}}-k_{a 1} \frac{\partial f_{g a}\left(\boldsymbol{x}_{i}, \boldsymbol{x}^{r}\right)}{\partial x_{i}}$

$\boldsymbol{F}_{i}^{2}=\boldsymbol{F}_{i}^{l a}+\boldsymbol{F}_{i}^{l r}=-\nabla U_{i}^{l a}-\nabla U_{i}^{l r}$

$=-k_{a 2} \sum_{j=1}^{N} \frac{\partial f_{l a}\left(\boldsymbol{x}_{i}, \boldsymbol{x}_{j}^{d}\right)}{\partial \boldsymbol{x}_{i}}-2 k_{r 2} \sum_{j=1, j \neq i}^{N} \frac{\partial f_{l r}\left(\boldsymbol{x}_{i}, \boldsymbol{x}_{j}\right)}{\partial \boldsymbol{x}_{i}}$

The last part of the controller is a virtual repulsive force generated by environmental constraints. Based on the potential field equation, we mentioned in section 3.5, we obtain the associated virtual force is:

$$
\begin{aligned}
& \begin{array}{l}
\boldsymbol{F}^{c}\left(\boldsymbol{x}_{i}\right)= \\
\quad=-\nabla U_{i}^{c} \\
\quad=\sum_{r=1}^{R} k_{c r} \iint_{-\infty}^{\infty} \frac{\delta\left(\left\|\boldsymbol{\Theta}_{i}\right\|\right)\left(\boldsymbol{x}_{i}-\boldsymbol{s}\right)}{\Psi_{i}^{2}\left\|\boldsymbol{x}_{\boldsymbol{i}}-\boldsymbol{s}\right\|} d s_{1} d s_{2}
\end{array} \\
& \boldsymbol{\Theta}_{\mathrm{i}}=\boldsymbol{x}_{i}-\boldsymbol{s}-k P_{r}(\boldsymbol{s}) \\
& \Psi_{\mathrm{i}}=\left\|\boldsymbol{x}_{i}-\boldsymbol{s}\right\|-s_{0}
\end{aligned}
$$

For a collision-free formation (as well as avoiding collisions with any external obstacles), we have to make sure that the potential field generated by a constrained space (an obstacle) should act only on the virtual leader (i.e. the formation center). This will locally modify the given trajectory of the virtual leader, and will help avoid collisions with the boundaries of activity area (the whole flying envelope of the UAVs) and any other external obstacles. The collision avoidance potential force on the virtual leader can now be written as: 
$\boldsymbol{F}^{c}\left(\boldsymbol{x}^{r}\right)=\sum_{r=1}^{R} k_{c r} \iint_{-\infty}^{\infty} \frac{\delta(\|\Theta\|)\left(\boldsymbol{x}^{r}-\boldsymbol{s}\right)}{\Psi^{2}\left\|\boldsymbol{x}^{r}-\boldsymbol{s}\right\|} d s_{1} d s_{2}$

$\boldsymbol{\Theta}=\boldsymbol{x}^{r}-\boldsymbol{s}-k P_{r}(\boldsymbol{s})$

$\Psi=\left\|\boldsymbol{x}^{r}-\boldsymbol{s}\right\|-s_{0}-\lambda$

Denote $\widetilde{\boldsymbol{x}}^{r}=\boldsymbol{x}^{r *}-\boldsymbol{x}^{r}$, where $\widetilde{\boldsymbol{x}}^{r}$ is the bias between the modified trajectory $\boldsymbol{x}^{r *}$ and the desired trajectory $\boldsymbol{x}^{r}$. The adaptive law for the modified trajectory is

$\dot{\boldsymbol{x}}^{r *}=\dot{\boldsymbol{x}}^{r}+\boldsymbol{F}^{c}\left(\boldsymbol{x}^{r}\right)-k_{t} \widetilde{\boldsymbol{x}}^{r}$

Overall, the controller can be rewritten as:

$\boldsymbol{u}_{i}= \begin{cases}\boldsymbol{G}_{i}^{+}\left(-\boldsymbol{F}_{i}+\boldsymbol{F}_{i}^{1}+\dot{\boldsymbol{x}}^{r *}\right), & \text { if } \text { Phase }=1 \\ \boldsymbol{G}_{i}^{+}\left(-\boldsymbol{F}_{i}+\boldsymbol{F}_{i}^{2}+\dot{\boldsymbol{x}}^{r *}\right), & \text { if Phase }=2\end{cases}$

The pseudo code for Modified Artificial Potential Field method showed as follows.

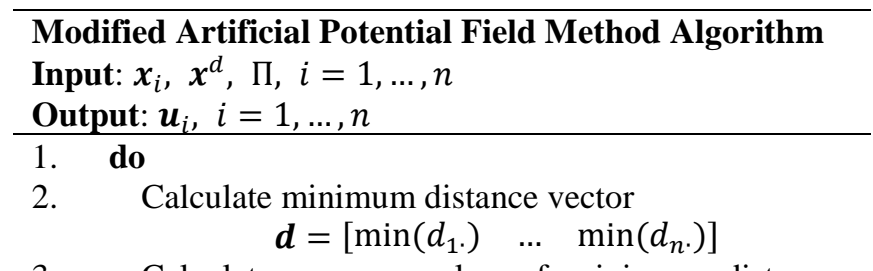

3. Calculate average value of minimum distances between UAVs

$$
\bar{d}=\frac{\min \left(d_{1} .\right)+\min \left(d_{2}\right)+\cdots+\min \left(d_{n} .\right)}{n}
$$

4. Calculate standard deviation of minimum distances between UAVs

$\sigma$

$=\sqrt{\frac{1}{n}\left[\left(\min \left(d_{1}\right)-\bar{d}\right)^{2}+\cdots+\left(\min \left(d_{n}\right)-\bar{d}\right)^{2}\right]}$

5. for $i=1, \ldots, n$

6. Calculate collision avoidance force $\boldsymbol{F}^{c}\left(\boldsymbol{x}^{r}\right)$

7. Calculate $\dot{\boldsymbol{x}}^{r *}=\dot{\boldsymbol{x}}^{r}+\boldsymbol{F}^{c}\left(\boldsymbol{x}^{r}\right)-k_{t} \widetilde{\boldsymbol{x}}^{r}$

8. $\quad$ if Phase $==1$

9. $\quad$ Calculate $\boldsymbol{F}_{i}^{\text {gat }}$

$10 \quad$ Calculate $\boldsymbol{u}_{i}=\boldsymbol{G}_{i}^{+}\left(-\boldsymbol{F}_{i}+\boldsymbol{F}_{i}^{1}+\dot{\boldsymbol{x}}^{r *}\right)$

end if

19 while formation_error $>$ tollerance

\section{FORMATION GENERATION SIMULATION STUDY}

To illustrate the feasibility of the design of formation generation, numerous 2D simulations are carried out, in which four UAVs are used to generate a rectangle formation from random positions. We simulated both of the original Formation Potential Field method (as suggested by Liu et al. [9]) and our proposed Modified Artificial Potential Field method, simultaneously in each 4-UAV set up. Some assumptions on the environment and parameters are considered as listed below:

(1) We assume all UAVs are linear time-invariant systems, and have the following form of dynamic properties:

$\dot{\boldsymbol{x}}_{i}=\left[\begin{array}{l}2 \\ 4\end{array}\right]+\left[\begin{array}{ll}2 & 0 \\ 0 & 2\end{array}\right] \boldsymbol{u}_{i}$

(2) The given formation positions are: $(-2,-2),(-2,2)$, $(2,-2),(2,2)$. Therefore $\lambda=\sqrt{2} / 2$.

(3) The global attractive potential parameter (19) $k_{a 1}=$ 0.99 .

(4) The ratio between the local repulsive potential parameter for Phase 1 with the global attractive potential is: $k_{r 1}=$ $6 \lambda^{2} k_{a 1}=2.97$.

(5) The local attractive potential parameter $k_{a 2}=0.99$.

(6) The local repulsive potential parameter for Phase 2 is: $k_{r 2}=k_{a 2} / 0.43=2.30$.

(7) The upper limit of input saturation is $S_{\max }=0.1$.

(8) Tolerance of tracking error is $\varepsilon=0.059$

In most cases, the FPF method and the MAPF method behaved as expected. But when randomly generated locations of UAVs are not well distributed in the space, the original FPF have a high chance fail to achieve its goal. Figure 7 shows an example when the FPF. At the beginning of the formation generation process, all UAVs are heading to the target position. Since $U A V_{2}, U A V_{3}$ and $U A V_{4}$ are nearer to the target locationfs (marked as blck crosses) than $\mathrm{UAV}_{1}$, these three UAVs will head towards the nearest target position and waiting there. But when $\mathrm{UAV}_{1}$ near to the target location, the local attractive potential field's influence will be neutralized by the other three UAVs' local repulsive potential fields and will result in a formation stalemate.

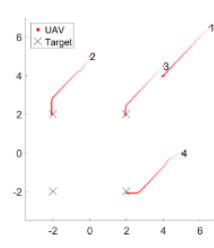

Figure 7. FPF formation generation failure

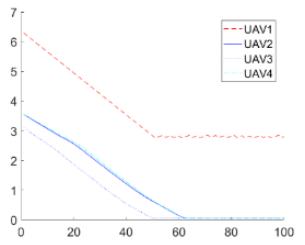

Figure 8. FPF formation generation error

Figure 8 shows the formation tracking error of each UAV. Just like what we described above, after around 50 time-units, 
$\mathrm{UAV}_{1}$ has lost its way to its target location and its tracking error will not be reduced anymore.

We have then our proposed MAPF method under the same set up. Figure 9 shows the trajectory of each UAV and how they generate the given formation. We can see that, at the beginning stage, all UAVs are heading to the formation area directly. When they come close enough to this area, they move around the formation center (which directly related with $k_{a 1} / k_{r 1}$ ) to make sure all UAVs are well distributed. Once Phase 1's goal is achieved, all UAVs will move toward to the nearest target location directly.

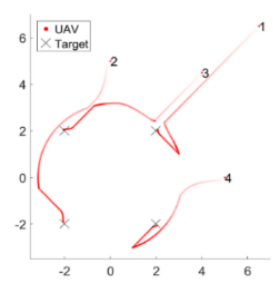

Figure 9. MAPF method formation generation

Figure 10 shows the tracking error of each UAV in both Phase 1 and Phase 2. At first, errors of all UAVs decrease quickly. After around 75 time-units, UAVs start moving around the formation center to make sure all UAVs come near to the formation area and spread well. Because of this kind of behavior, the tracking error goes up a little bit. And at the last stage, all UAVs move towards the target position as expected.

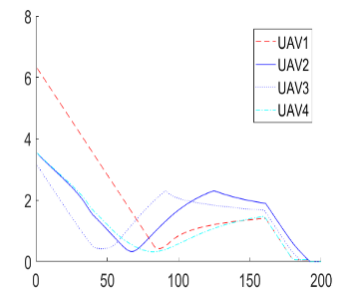

Figure 10. MAPF formation generation error

\section{CONCLUSIONS}

In this paper, the Modified Artificial Potential Field method has been proposed to control a group of autonomous UAVs to achieve and maintain a given formation while avoiding collisions. Unlike other centralized UAV formation control methods, the MAPF method does not require high computational capability and the flying trajectory can be modified in real time when an unexpected obstacle has been detected. The MAPF method has two phases. UAVs will be gathered around the formation center during Phase 1 and the given formation will be achieved in Phase 2. Simulation results show the capability of the algorithm dealing with formation processing.

\section{ACKNOWLEDGMENT}

This work has been partially funded by the Center for Advanced Systems and Engineering (CASE) at Syracuse University and Gryphon Sensors.

\section{REFERENCES}

[1] Casbeer D.W., Li S.M., Beard R.W., McLain T.W., Mehra R.K. (2005). Forest fire monitoring using multiple small UAVs, American Control Conference, Vol. 5, pp. 3530-3535.

[2] Maithripala D.H.A., Jayasuriya S. (2005). Radar deception through phantom track generation, $A C C$, DOI: $10.1109 /$ ACC. 2005.1470620

[3] Khatib O. (1986). Real-time obstacle avoidance for manipulators and mobile robots, The International Journal of Robotics Research, Vol. 5, No. 1, pp. 90-98. DOI: $10.1177 / 027836498600500106$

[4] Laumond J.P. (1998). Robot motion planning and control, Springer. DOI: 10.1007/BFb0036069

[5] Hussien B. (1989). Robot path planning and obstacle avoidance by means of potential function method, $\mathrm{Ph}$. D Dissertation, University of Missouri-Columbia, USA.

[6] Ge S.S., Cui Y.J. (2000). New potential functions for mobile robot path planning, IEEE Transactions on Robotics and Automation, Vol. 16, No. 5, pp. 615-620. DOI: $\underline{10.1109 / 70.880813}$

[7] Yang Y., Wang S., Wu Z., Wang Y. (2011). Motion planning for multi-HUG formation in an environment with obstacles, Ocean Engineering, Vol. 38, No. 17, pp 2262-2269. DOI: 10.1016/j.oceaneng.2011.10.008

[8] Asl A.N., Menhaj M.B., Sajedin A. (2014). Control of leader-follower formation and path planning of mobile robots using Asexual Reproduction Optimization (ARO), Applied Soft Computing, Vol. 14, pp. 563-576.

[9] Liu X., Ge S.S., Goh C.H. (2016). Formation potential field for trajectory tracking control of multi-agentsin constrained space, International Journal of Control, pp $1-15$.

[10] Wei E., Li T., Hu Y. (2013). Robust adaptive neural network control for wheeled inverted pendulum with input saturation, 10th International Symposium on Neural Networks, Dalian. DOI: 10.1007/978-3-64239068-5 6

[11] Ge S.S., Liu X., Goh C.H., Xu L. (2015). Formation tracking control multi-agents in constrained space, IEEE Transactions on Control Systems Technology, Vol. 24, No. 3, pp. 992-1003.

[12] Chen M., Ge S.S., Choo Y.S. (2009). Neural network tracking control of ocean surface vessels with input saturation, The IEEE International Conference on Automation and Logistics, Shenyang, China. DOI: 10.1109/ICAL.2009.5262972

[13] Ma H., Wang M., Jia Z., Yang C. (2011). A new framework of optimal multi-robot formation, 30th Chinese Control Conference.

[14] Wang X., Yadav V., Balakrishanan S.N. (2007). Cooperative UAV formation flying with obstacle/collision avoidance, IEEE Transactions on Control System Technology, Vol. 15, No. 4, pp. 672679. DOI: $10.1109 /$ TCST.2007.899191

[15] Luo G., Yu J., Mei Y., Zhang S. (2015). UAV path planning in mixed-obstacle environment via artificial potential field method improved by additional control force, Asian Journal of Control, Vol. 17, No. 5, pp. 1600-1610. 